\title{
Multinuclear Spin-Lattice Relaxation Time Studies of Supercooled Aqueous LiCl-Solutions
}

\author{
E. W. Lang and F. X. Prielmeier \\ Institut für Biophysik und physikalische Biochemie, Universität Regensburg, \\ Postfach 317, D-8400 Regensburg, Federal Republic of Germany
}

\section{Diffusion / Liquids / Solutions / Spectroscopy, Nuclear Magnetic Resonance / Transport Properties}

Recent deuteron $\left({ }^{2} \mathrm{H}\right)$ spin-lattice relaxation time $\left(T_{1}\right)$ measurements in undercooled $11 \mathrm{~m} \mathrm{LiCl} / \mathrm{D}_{2} \mathrm{O}$ solutions are supplemented by proton $\left({ }^{1} \mathrm{H}\right)$ and lithium $\left({ }^{6} \mathrm{Li}\right)-T_{1}$ as well as self-diffusion coefficient measurements in undercooled $11 \mathrm{~m} \mathrm{LiCl} / \mathrm{H}_{2} \mathrm{O}$ solutions. The motional model for orientational fluctuations, proposed recently to interpret the ${ }^{2} \mathrm{H}-T_{1}$ experiments, is slightly modified and is shown to describe the relaxation time curves of all nuclei $\left({ }^{2} \mathrm{H},{ }^{1} \mathrm{H},{ }^{6} \mathrm{Li}\right)$ with only one adjustable parameter for light and heavy water. Self-diffusion coefficients serve to calculate the contribution to the relaxation rates from positional fluctuations of the spin-bearing particles. It is found that $D\left({ }^{1} \mathrm{H}\right)>D\left({ }^{7} \mathrm{Li}\right)$ and that the proton mobility is less strongly slowed down when the temperature is lowered towards the glass temperature. Possible relations between this excess proton mobility and a recently observed excess proton conductivity are discussed.

\section{Introduction}

The physical properties of aqueous LiCl-solutions have been studied by a variety of experimental techniques in the past. Recently the molecular motions of water molecules could be investigated in strongly undercooled LiCl-solutions in the composition range $4.5<C\left(=\right.$ moles $\mathrm{H}_{2} \mathrm{O} /$ mole $\left.\mathrm{LiCl}\right)$ $<\infty$ via ${ }^{2} \mathrm{H}$ spin-lattice relaxation time $\left(T_{1}\right)$ measurements $[1-3]$. At low temperatures molecular motions are slowed down and the observed relaxation rates $\left(R_{1}=1 / T_{1}\right)$ become sensitive to the details of the molecular dynamics. A simple motional model has been developed for water molecules hydrating the cations in a solution with composition $C=4.55$ and a two-state assumption was sufficient to calculate the relaxation rate curves $R_{1}(T)$ for all compositions $C>4.55$. In the present investigation ${ }^{1} \mathrm{H}$ and ${ }^{6} \mathrm{Li}$ relaxation time as well as self-diffusion coefficient measurements in $11 \mathrm{~m} \mathrm{LiCl} / \mathrm{H}_{2} \mathrm{O}$ solutions are reported. Both nuclei relax mainly via magnetic dipole-dipole interactions [4]. The latter are modulated by orientational and positional fluctuations of the spin-bearing particles. Hence, whereas ${ }^{2} \mathrm{H}$ relaxation rates monitor single particle orientational fluctuations only, rotational and translational diffusive modes determine the relaxation rates of ${ }^{1} \mathrm{H}$ and ${ }^{6} \mathrm{Li}$-nuclei. Self-diffusion coefficient measurements provide a direct measure of the positional fluctuations in the system and allow the calculation of the contribution of the translational diffusive modes to the observed relaxation rates.

\section{Experiment}

The solutions were prepared from a stock solution of the anhydrous salt (Merck, Darmstadt, Suprapur) and $\mathrm{D}_{2} \mathrm{O}(99.95 \%$, Merck, Darmstadt). Prior to use, the samples have been degassed in a $5 \mathrm{~mm}$ NMR sample tube by at least five freeze-pumpe-thaw cycles to remove dissolved oxygen. All $T_{1}$-measurements were obtained with the inversion recovery pulse sequence with alternating phases on a 
Bruker MSL-300 spectrometer $\left(B_{0}=7.05\right.$ Tesla). They are considered reliable to $\pm 10 \%$. The self-diffusion coefficient measurements were done with a home-built probe with Helmholtz-coils providing the pulsed field-gradient. Details of the probe have been published elsewhere [5]. The self-diffusion coefficients are accurate to $\pm 5 \%$ except for the lowest temperatures where the accuracy degrades to $\leqslant 10 \%$. The temperatures were measured with a miniature chromelalumel thermocouple (Philips, Kassel) and are accurate to $\pm 1 \mathrm{~K}$.

\section{Theory}

A wealth of information concerning the average local structure of the ion - water arrangement and the water molecule geometry in concentrated aqueous $\mathrm{LiCl}$-solutions is available from neutron - and X-ray scattering [6-9], NMR [10 - 12], MD [13,14]- and MC [15, 16]-simulations. These experiments confirm a well-defined coordination shell for $\mathrm{Li}^{+}$and provide accurate internuclear distances. Accordingly water molecules are oriented relative to a $\mathrm{Li}^{+}$cation with their protons pointing away from the cation and with their dipole moment vector deviating from the radial direction by $52 \mathrm{deg}$. in concentrated solutions. Of course, any model of the dynamics of water molecules has to be consistent with the average local structure in these solutions. Hence the model of the orientational fluctuations that will be applied considers water molecules adjacent to a strongly hydrating cation to execute small-amplitude librations superimposed onto anisotropic orientational fluctuations about the local director (ion-oxygen distance). In addition the molecules diffuse around the ion and chemical exchange occurs. The tumbling of the water molecules around the ion does not necessarily imply the rotation of a rigid hydration complex on the time-scale of the mean residence time $\tau_{\mathrm{ex}}$ of a water molecule in the neighbourhood of an ion. Rather the isotropic tumbling around the ion results from collective configurational fluctuations associated with the glass transition $[2,17]$.

Theoretical expressions for the relaxation rates have been worked out for electric quadrupole interactions $[2,3]$ but may be obtained for magnetic dipole interactions in a straightforward manner. For ${ }^{1} \mathrm{H}$-nuclei the pertinent relaxation rate may be decomposed into an intramolecular and an intermolecular contribution due to the dipolar coupling to the second proton within the water molecule and to all other protons as well as to the nuclei ${ }^{7} \mathrm{Li}(92.58 \%)$ and ${ }^{6} \mathrm{Li}$ $(7.42 \%)$.

$R_{1}(1 \mathrm{H})=R_{1, \text { intra }}(1 \mathrm{H})+R_{1, \text { inter }}(1 \mathrm{H})$

with

$$
\begin{aligned}
R_{1, \text { intra }}(1 \mathrm{H})= & R_{1, \mathrm{OR}}(1 \mathrm{H}-1 \mathrm{H}) \\
& +0.9258 \cdot R_{1, \mathrm{OR}}(1 \mathrm{H}-7 \mathrm{Li}) \\
& +0.0742 \cdot R_{1, \mathrm{OR}}(1 \mathrm{H}-6 \mathrm{Li})
\end{aligned}
$$

and

$R_{1, \text { inter }}(1 \mathrm{H})=R_{1, \text { POS }}(1 \mathrm{H}-1 \mathrm{H})+R_{1, \text { POS }}(1 \mathrm{H}-7 \mathrm{Li})$.

In general dipolar relaxation rates between like spins $\left(I, I^{\prime}\right)$ and unlike spins $(I, S)$ are given by
$R_{1}\left(I, I^{\prime}\right)=(1 / 5)\left(\gamma_{l} \gamma_{l} h / 2 \pi\right)^{2} I(I+1)\left[2 \cdot J\left(\omega_{l}\right)+8 \cdot J\left(2 \omega_{l}\right)\right]$

and

$$
\begin{aligned}
R_{1}(I, S)= & (2 / 15)\left(\gamma_{I} \gamma_{S} h / 2 \pi\right)^{2} S(S+1) \\
& \cdot\left[J\left(\omega_{I}-\omega_{S}\right)+3 \cdot J\left(\omega_{l}\right)+6 \cdot J\left(\omega_{I}+\omega_{S}\right)\right]
\end{aligned}
$$

respectively with $J(\omega)$ the spectral density function of the relevant fluctuating variable $[2,3]$. The intramolecular contribution monitors orientational fluctuations only. The rotational propagator according to the motional model has been obtained recently [2] and may be applied yielding

$$
\begin{aligned}
R_{1, \text { OR }}(1 \mathrm{H}-1 \mathrm{H})= & \langle\mathrm{COR}(1 \mathrm{H}-1 \mathrm{H})\rangle \\
& \cdot\left[(1 / 4)\left(3 \cos ^{2} \beta-1\right)^{2} \cdot F\left(\omega_{\mathrm{H}} \tau_{0}\right)\right. \\
& \left.+(3 / 4) \sin ^{4} \beta \cdot F\left(\omega_{\mathrm{H}} \tau_{2}\right)\right] \\
R_{1, \mathrm{OR}}(1 \mathrm{H}-7 \mathrm{Li})= & \langle\mathrm{COR}(1 \mathrm{H}-7 \mathrm{Li})\rangle \\
& \cdot\left[(1 / 4)\left(3 \cos ^{2} \beta-1\right)^{2} \cdot F\left(\omega_{\mathrm{H}} \tau_{0}, \varepsilon_{1}\right)\right. \\
& +3\left(\sin \beta \cos ^{2} \beta\right)^{2} \cdot F\left(\omega_{\mathrm{H}} \tau_{1}\right) \\
& \left.+(3 / 4) \sin ^{4} \beta \cdot F\left(\omega_{\mathrm{HI}} \tau_{2}, \varepsilon_{1}\right)\right] \\
R_{1, \mathrm{OR}}(1 \mathrm{H}-6 \mathrm{Li})= & \langle\mathrm{COR}(1 \mathrm{H}-6 \mathrm{Li})\rangle \\
& \cdot\left[(1 / 4)\left(3 \cos ^{2} \beta-1\right)^{2} \cdot F\left(\omega_{\mathrm{II}} \tau_{0}, \varepsilon_{2}\right)\right. \\
& +\left(\sin \beta \cos ^{2} \beta\right)^{2} \cdot F\left(\omega_{\mathrm{H}} \tau_{1}\right) \\
& \left.+(3 / 4) \sin ^{4} \beta \cdot F\left(\omega_{1 \mathrm{II}} \tau_{2}, \varepsilon_{1}\right)\right]
\end{aligned}
$$

with

$$
\begin{aligned}
\langle\operatorname{COR}(1 \mathrm{H}-1 \mathrm{H})\rangle= & (3 / 10)\left[\gamma_{\mathrm{H}} \gamma_{\mathrm{H}}(h / 2 \pi)\right. \\
& \left.\cdot\left\langle\left(r_{\mathrm{HH}}\right)^{-3}\right\rangle\left(\mu_{0} / 4 \pi\right)^{2}\right]^{2} \\
\langle\operatorname{COR}(1 \mathrm{H}-7 \mathrm{Li})\rangle= & (1 / 2)\left[\gamma_{\mathrm{H}} \gamma_{7 \mathrm{Li}}(h / 2 \pi)\right. \\
& \left.\cdot\left\langle\left(r_{\mathrm{HLi}}\right)^{-3}\right\rangle\left(\mu_{0} / 4 \pi\right)\right]^{2} \\
\langle\operatorname{COR}(1 \mathrm{H}-6 \mathrm{Li})\rangle= & (4 / 15)\left[\gamma_{\mathrm{H}} \gamma_{6 \mathrm{Li}}(h / 2 \pi)\right. \\
& \left.\cdot\left\langle\left(r_{\mathrm{HLi}}\right)^{-3}\right\rangle\left(\mu_{0} / 4 \pi\right)\right]^{2}
\end{aligned}
$$

Whereas the slower diffusive modes contribute to the spec tral density function, the effect of the fast torsional oscillations is incorporated into an effective, librationally averaged coupling constant $\langle\operatorname{COR}(I-S)\rangle[3,18]$. The angle $\beta$ meas ures the deviation of the vector connecting the nuclei $I$ and $S$ from the local director. With the local structure of the ion-water arrangement as deduced from neutron scattering results [6] an angle $\beta=90 \mathrm{deg}$. is obtained for ${ }^{1} \mathrm{H}-{ }^{1} \mathrm{H}$ interactions and $\beta=21 \mathrm{deg}$. for $\mathrm{Li}^{-1} \mathrm{H}$ interactions. The corresponding internuclear distances $r_{\mathrm{HH}}$ and $r_{\mathrm{HLi}}$ are taken from NMR [10]- and neutron scattering experiments [6] and are compiled in Table 1 . Concerning the $\mathrm{Li}-\mathrm{H}$ correlations there is some scatter in the data obtained from var ious sources [14]. $F(\omega \tau)$ and $F(\omega \tau, \varepsilon)$ are given by the terms in brackets in Eqs. (2) and (3) respectively with 
Table 1

Compilation of model parameters entering the relaxation rate expressions

\begin{tabular}{|c|c|c|c|c|}
\hline Parameter & & $\mathrm{H}_{2} \mathrm{O}$ & $\mathrm{D}_{2} \mathrm{O}$ & Ref. \\
\hline$\tau_{0)} \quad(p s)$ & & 0.200 & 0.200 & \\
\hline$\tau_{i 0} \quad(p s)$ & & 0.059 & 0.080 & \\
\hline$E_{\mathrm{a}} \quad(\mathrm{kJ} / \mathrm{mol})$ & & 18.71 & 19.79 & \\
\hline$B_{\mathrm{r}} \quad(\mathrm{kJ} / \mathrm{mol})$ & & 5.72 & 5.72 & \\
\hline \multirow[t]{2}{*}{$B_{\mathrm{f}} \quad(\mathrm{kJ} / \mathrm{mol})$} & 'H & 5.26 & & \\
\hline & ${ }^{7} \mathrm{Li}$ & 5.58 & & \\
\hline \multirow[t]{2}{*}{$D_{0} \quad\left(\mathrm{~m}^{2} / \mathrm{s}\right)$} & ${ }^{1} \mathrm{H}$ & $2.54 \cdot 10^{-8}$ & & \\
\hline & ${ }^{7} \mathrm{Li}$ & $1.91 \cdot 10^{-8}$ & & \\
\hline$\%$ eff $\quad(k H z)$ & ${ }^{2} \mathrm{H}$ & & 192.0 & \\
\hline$r_{\mathrm{HH}}(\mathrm{nm})$ & & 0.159 & & {$[10]$} \\
\hline$r_{\mathrm{HLi}}(\mathrm{nm})$ & & 0.250 & & [6] \\
\hline$d_{\mathrm{HH}}(\mathrm{nm})$ & & 0.225 & & [14] \\
\hline$d_{\mathrm{H} 1, i}(\mathrm{~nm})$ & & 0.335 & & [14] \\
\hline
\end{tabular}

$J(\omega)=\tau /\left(1+(\omega \tau)^{2}\right)$

Also

$\varepsilon_{1}=\omega_{7 \mathrm{Li}} / \omega_{\mathrm{II}}, \quad \varepsilon_{2}=\omega_{6 \mathrm{Li}} / \omega_{\mathrm{H}}$

$1 / \tau_{0}=1 / \tau_{\mathrm{r}}+1 / \tau_{\mathrm{ex}}$

$1 / \tau_{1}=1 / \tau_{0}+1 / \tau_{\mathrm{i}}$

$1 / \tau_{2}=1 / \tau_{0}+4 / \tau_{i}$

In deducing these expressions it had to be assumed that time-scale separation pertains between the torsional and the diffusive modes, that the anisotropic diffusive mode with correlation time $\tau_{i}$ and the isotropic overall tumbling mode with correlation time $\tau_{\mathrm{r}}$ are statistically independent, that the mean residence time $\tau_{\mathrm{cx}}$ is at least of the order of the correlation times $\tau_{\mathrm{r}}, \tau_{\mathrm{i}}$, i.e. $\tau_{\mathrm{r}}, \tau_{\mathrm{j}}<\tau_{\mathrm{ex}}$, and that the interaction is completely randomized on exchange [19]. This should be a reasonable assumption for small molecules providing the perturbing surface because of the vanishingly small probability for surface reencounters within $\tau_{\mathrm{r}}$ [20]. Further it is important to realize that an exponential decay of the orientational correlation function for times $t>\tau_{\mathrm{r}}$ is implied by Eq. (7), though non-exponential correlation functions are commonly observed in undercooled systems near their glass transition temperatures [21]. However, data at different fields $B_{0}$ and at very low temperatures corresponding to the slow motions regime $(\omega \tau>1)$ are necessary to differentiate between a Debye- and a Kohlrausch law [22,23]. The ${ }^{2} \mathrm{H}-T_{1}$ could not be followed to low enough temperatures at 2.35 Tesla to allow a decision between both relaxation laws. Although the spin quantum number of the ${ }^{6} \mathrm{Li}$-nucleus is $I=1$ it has all virtues of a spin $1 / 2$ nucleus due to the extremely small electric quadrupole moment $[24,25]$. Hence the spin-lattice coupling is mediated predominantly via magnetic dipole interactions. Due to the well-defined hydration sphere of the $\mathrm{Li}^{+}$-cation together with a reasonably long mean residence time of the water molecules in the coordination shell [26] the dipolar interaction of the ${ }^{6} \mathrm{Li}$-nucleus with the protons of the adjacent water molecules is assumed to be modulated by orientational fluctuations only with constant average distance $r_{\mathrm{Li}-\mathrm{H}}$. The corresponding relaxation rate $R_{1 . \text { OR }}(6 \mathrm{Li}-1 \mathrm{H})$ may be obtained from Eq. (6) by interchanging $\omega_{\mathrm{H}}$ and $\omega_{6 \mathrm{Li}}$ and replacing $\langle\mathrm{COR}(1 \mathrm{H}-$ 6Li) $>$ by

$$
\begin{aligned}
\langle\operatorname{COR}(6 \mathrm{Li}-1 \mathrm{H})\rangle= & (1 / 10) \cdot\left[\gamma_{\mathrm{H}} \gamma_{6 \mathrm{Li}}(h / 2 \pi)\right. \\
& \left.\cdot\left\langle\left(r_{\mathrm{LiH}}\right)^{-3}\right\rangle\left(\mu_{0} / 4 \pi\right)\right]^{2} \cdot n_{\mathrm{HH}}
\end{aligned}
$$

with $n_{\mathrm{H}}=8$ the number of nearest neighbour protons.

Intermolecular dipolar interactions are to a large extent modulated by translational motions of the spin-bearing particles. The translational propagator needed to calculate the corresponding correlation function of the fluctuating variable is assumed to obey a force-free diffusion equation with reflecting boundary conditions $[27,28]$. Any pair-correlation effects are neglected [29] as well as a coupling of rotational and translational motions [30]. The intermolecular relaxation rates of Eq. $(1 \mathrm{~b})$ are given by

$$
\begin{aligned}
& R_{1, \operatorname{POS}}(1 \mathrm{H}-1 \mathrm{H})=\operatorname{CPOS}(1 \mathrm{H}-1 \mathrm{H}) \cdot F\left(\omega_{\mathrm{H}} \tau_{\mathrm{d}}\right) \\
& R_{1, \operatorname{POS}}(1 \mathrm{H}-7 \mathrm{Li})=\operatorname{CPOS}(1 \mathrm{H}-7 \mathrm{Li}) \cdot F\left(\omega_{\mathrm{H}} \tau_{\mathrm{d}}, \varepsilon_{1}\right)
\end{aligned}
$$

with

$$
\begin{aligned}
& \operatorname{CPOS}(1 \mathrm{H}-1 \mathrm{H})=(6 \pi / 5) \cdot\left[\gamma_{1 \mathrm{H}}^{2}\left(h \cdot \mu_{0} / 8 \pi\right)\right]^{2} \\
& \operatorname{CPOS}(1 \mathrm{H}-7 \mathrm{Li})=2 \pi \cdot\left[\gamma_{\mathrm{H}} \gamma_{\mathrm{Li}}\left(h \cdot \mu_{0} / 8 \pi\right)\right]^{2} .
\end{aligned}
$$

$F(\omega \tau)$ and $F\left(\omega \tau, \varepsilon_{1}\right)$ are again given by the term in brackets in Eqs. (2) and (3) with

$$
\begin{aligned}
J(\omega)= & \left(N_{\mathrm{s}} \cdot \tau_{\mathrm{d}} / d_{I-s}^{3}\right) \cdot\left[\left((3 / 2) \cdot u^{2}\right.\right. \\
& +(15 / 2) \cdot u+12) \cdot\left((1 / 8) \cdot u^{6}+u^{5}+4 \cdot u^{4}\right. \\
& \left.\left.+(27 / 2) \cdot u^{3}+(81 / 2) \cdot u^{2}+81 \cdot u+81\right)^{-1}\right]
\end{aligned}
$$

whereby

$u=\left(2 \omega \tau_{\mathrm{d}}\right)^{(1 / 2)}$ and $\tau_{\mathrm{d}}=d_{I-S}^{2} / D_{\mathrm{rcl}}(I-S)$

and

$D_{\text {rel }}(1 \mathrm{H}-1 \mathrm{H})=2 \cdot D_{\text {self }}((1 \mathrm{H})$

$D_{\text {rcl }}(1 \mathrm{H}-7 \mathrm{Li})=D_{\text {self }}(1 \mathrm{H})+D_{\text {self }}(\mathrm{Li})$

$d_{\mathrm{I}-\mathrm{S}}$ is the distance of closest approach of the spin-bearing particles and $D_{\text {sclf }}(\mathrm{X})$ the experimentally determined selfdiffusion coefficient of the species $\mathrm{X}$. The contribution from positional fluctuations to the ${ }^{6} \mathrm{Li}$ relaxation rate $R_{1, \operatorname{POS}}(6 \mathrm{Li}-1 \mathrm{H})$ is given accordingly by

$$
R_{1, \operatorname{POS}}(6 \mathrm{Li}-1 \mathrm{H})=\operatorname{CPOS}(6 \mathrm{Li}-1 \mathrm{H}) \cdot F\left(\omega_{\mathrm{Li}} \tau_{\mathrm{d}}, \varepsilon_{2}^{-1}\right)
$$

with

$\operatorname{CPOS}(6 \mathrm{Li}-1 \mathrm{H})=(2 \pi / 5) \cdot\left[\gamma_{6 \mathrm{Li}} \gamma_{\mathrm{H}} h\left(\mu_{0} / 4 \pi\right)\right]^{2}$ 


\section{Results and Discussion}

During the last years we had been interested in the anomalous physical properties of undercooled water resulting from the build-up of long-range structural correlations within the random transient hydrogen-bonded network. These cooperative phenomena imply an increasing correlation length and a strong slowing down of structural fluctuations with decreasing temperature. To that end spin-lattice relaxation times of ${ }^{1} \mathrm{H},{ }^{2} \mathrm{H}$ and ${ }^{17} \mathrm{O}$ have been measured to study the molecular motions of water molecules in the metastable phase and to investigate how these motions become modified by the addition of network breaking agents like hydrostatic pressure or ionic solutes $[3,31]$. It has been shown that a destruction of the $\mathrm{H}$-bond network suppresses long-range density-density correlations and turns water into a normal viscous liquid which then can be undercooled quite substantially. This is especially true for highly concentrated aqueous electrolyte solutions which can be undercooled to their respective glass-transition temperatures. At these temperatures the observed spin-lattice relaxation rates become sensitive to the details of the molecular motions. Hence models of the molecular dynamics, generally necessary to interpret NMR-relaxation rate curves, can be tested more thoroughly and structural parameters deduced.

While investigating the dynamic properties of water molecules in undercooled $\mathrm{LiCl}-\mathrm{D}_{2} \mathrm{O}$ solutions by ${ }^{1} \mathrm{H}-T_{1}$ measurements [2] advantage has been taken of the fact that in a solution of composition $C=4.55$ all water molecules may be considered to belong to the hydration sphere of the $\mathrm{Li}^{+}$cation. Consistent with the local structure of the $\mathrm{Li}^{+}\left(\mathrm{H}_{2} \mathrm{O}\right)_{n} \mathrm{Cl}^{-}$clusters as deduced from solid-state NMR [10] of the glassy phase and in accord with the assertion that the average local structures in the glass and in the undercooled solution are closely related, the simplest possible model has been developed and applied successfully to interpret ${ }^{2} \mathrm{H}-T_{1}$ data. Two earlier investigations of highly concentrated $\mathrm{LiCl}$-solutions were either not in accord with structural details of the hydration complex $[32,33]$ or resorted to empirical distribution functions of microscopic time constants characterizing the dynamics of water molecules in the clusters $[10,17]$. Again both models posses a very different frequency dependence in the slow motions regime. Although the applied model was simple with only a minimum of adjustable parameters and prooved adequate to describe the ${ }^{2} \mathrm{H}-T_{1}$ in all undercooled alkali-halide solutions with composition $C>4[2,3]$, some features of the model need further experimental test.

\section{Interpretation of the Model Parameters}

In our earlier investigations it turned out that the ${ }^{2} \mathrm{H}-T_{1}$ are not very sensitive to the details of the orientation of a water molecule relative to the $\mathrm{Li}^{+}$-cation, i.e. whether on average a trigonal or a tetragonal configuration applies. This is because the angle $\beta_{\mathrm{DF}}$ [2] between the local director and the mean OD-bond direction changes by only $16 \mathrm{deg}$. in going from the trigonal to the tetragonal configuration. Thus the tetragonal configuration has been assumed following neutron scattering results [6]. It seems to be the preferred orientation in most crystal hydrates also [34]. As regards the dependence of the $T_{1}(T)$ curve on the angle $\beta_{\mathrm{DF}}$ ${ }^{1} \mathrm{H}$ and ${ }^{6} \mathrm{Li}-T_{1}$ data do not provide a better probe because for ${ }^{1} \mathrm{H}-{ }^{1} \mathrm{H}$ interactions $\beta_{\mathrm{DF}}=90 \mathrm{deg}$. irrespective of the tilt angle $\Theta$ and for $\mathrm{Li}-\mathrm{H}$ interactions $\delta \beta_{\mathrm{DF}}=4 \mathrm{deg}$. between trigonal and tetragonal configurations. But dipolar interactions depend on the internuclear distances via $\left(1 / r_{I S}\right)^{6}$ hence are very sensitive to small variations of $r_{1 s}$.

Also the temperature dependence of the correlation time of the tumbling mode $\tau_{\mathrm{r}}$ and of the anisotropic internal mode $\tau_{\mathrm{i}}$ were unknown a priori. Guided by the shape of the deuterium relaxation rate curve $R_{1}(T)$ with its strong non-Arrhenius $T$-dependence in the fast motions regime at high temperatures, the seemingly Arrhenius behaviour at low temperatures and the glass-forming ability of these solutions led to the suggestion that the $T$-dependence of the relaxation rate is dominated at high temperatures by a Vogel-Tammann-Fulcher (VTF)-type [2] behaviour of the tumbling mode represented by

$\tau_{\mathrm{r}}=\tau_{\mathrm{r} 0} \exp \left(B_{\mathrm{r}} /\left(T-T_{0}\right)\right)$

with $B_{\mathrm{r}}$ an apparent activation energy of the collective structural rearrangements and $T_{0}$ the transition temperature of global motional arrest. Though signifying kinetic localisation, it should be closely related to the Kauzmann temperature $T_{\mathrm{K}}$, where the entropy of the liquid and the solid phases of the system would match.

According to this law the relaxation rate should slow down strongly at low temperatures contrary to what is actually observed. Hence, within this two-mode approximation, another mode with a much weaker $T$-dependence must take over at low temperatures and dominate the relaxation. This local anisotropic mode $\tau_{i}$ is considered a thermally activated process with its concomittant Arrhenius-type behaviour

$\tau_{\mathrm{i}}=\tau_{\mathrm{i} 0} \exp \left(E_{\mathrm{i}} / k T\right)$

It may resemble those local modes which are commonly thought to be responsible for the $\beta$-relaxation in glassy systems and which are still active below the glass-transition temperature $T_{0}$ [35]. Further it had been assumed earlier that $\tau_{\mathrm{cx}} \gg \tau_{\mathrm{r}}, \tau_{\mathrm{i}}$ thereby neclecting $\tau_{\mathrm{cx}}$, although with the weaker condition $\tau_{\mathrm{ex}} \geqslant \tau_{\mathrm{r}}, \tau_{\mathrm{i}}$ Eqs. (4) - (11) are still valid [19]. Not to introduce further unknowns and because only $1 / \tau_{0}=1 / \tau_{\mathrm{r}}+1 / \tau_{\mathrm{ex}}$ enters, the reasonable suggestion will be made that $\tau_{\mathrm{ex}}(T)$ also follows a VTF-law giving

$\tau_{0}=\tau_{00} \cdot \exp \left(B_{\mathrm{r}} /\left(T-T_{0}\right)\right)$

\section{Estimation of the Parameters for Orientational Fluctuations}

But how to cope with the various parameters in these equations? In the previous treatment [2] it has been explained how to estimate $B_{\mathrm{r}}$. Regarding $T_{0}$, a correlation between experimentally determined glass temperatures $T_{\mathrm{g}}$ and temperatures of minimum relaxation time $T_{\min }$ has been noted in this and other systems $[1-3]$ and used to predict the temperature of global motional arrest $T_{0}$. As experi- 


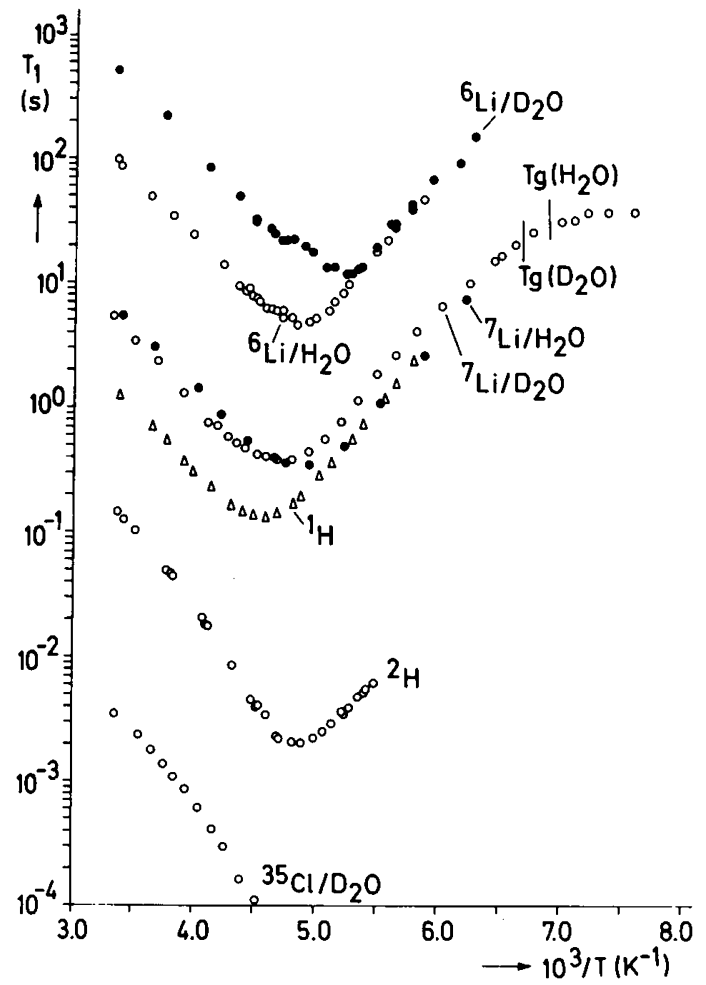

Fig. 1

Temperature dependence of the relaxation times of the nuclei ${ }^{35} \mathrm{Cl}$, ${ }^{2} \mathrm{H},{ }^{1} \mathrm{H},{ }^{6} \mathrm{Li}$ and effective relaxation times of ${ }^{7} \mathrm{Li}$ in aqueous $\mathrm{LiCl}$ solutions $\left(c=11 \mathrm{~m}, B_{0}=7.05\right.$ Tesla). $T_{\mathrm{g}}=$ glass temperature

mental glass temperatures $T_{\mathrm{g}}$ show an isotope effect of $\delta T_{\mathrm{g}}=4 \mathrm{~K}[36]$ in going from $\mathrm{D}_{2} \mathrm{O}$ to $\mathrm{H}_{2} \mathrm{O}$ in this system, $T_{\min }$ should show a corresponding isotope shift. This is indeed observed as shown in Fig. 1 where the spin-lattice relaxation time curves $T_{1}(T)$ of the nuclei ${ }^{35} \mathrm{Cl},{ }^{2} \mathrm{H},{ }^{1} \mathrm{H},{ }^{6} \mathrm{Li}$ and effective relaxation times $T_{1}(T)$ of ${ }^{7} \mathrm{Li}$, measured at $B_{0}=7$ Tesla, are drawn. For a direct comparison of the ${ }^{2} \mathrm{H}-$ $T_{1}$ and ' $\mathrm{H}-T_{1}$ complications arise because of different resonance frequencies of both nuclei. The isotope shift is most clearly seen in the $T_{1}(T)$ curves of ${ }^{7} \mathrm{Li}$ obtained in $\mathrm{D}_{2} \mathrm{O}$ and $\mathrm{H}_{2} \mathrm{O}$, where $\delta T_{\min }=4 \mathrm{~K}$. Besides both curves become congruent at temperatures $T>T_{\min }$ in a modified Arrhenius diagram $\ln \left(T_{1}\right)$ versus $\left(T-T_{0}\right)^{-1}$ demonstrating the absence of magnetic dipole interactions between ${ }^{7} \mathrm{Li}$ and protons or deuterons $[33,37]$. Thus the electric quadrupole interaction dominates completely implying large fluctuations of the electric field gradient (efg) at the ion $[38,39]$, hence of the symmetry of the proton arrangement within the hydration shell. Because the relaxation time minima of the ${ }^{7} \mathrm{Li}-T_{1}$ data occur at similar temperatures as for the water nuclei the ${ }^{7} \mathrm{Li}$-efg fluctuations are also driven by those motional modes leading to the relaxation of ${ }^{1} \mathrm{H},{ }^{2} \mathrm{H}$ and ${ }^{6} \mathrm{Li}[37]$. These aspects, however, will be dealt with in a subsequent paper [40]. Nevertheless these correlations predict $T_{0}=133 \mathrm{~K}$ in the 11 molal $\mathrm{LiCl} / \mathrm{H}_{2} \mathrm{O}$ solution.

The preexponential factor $\tau_{00}$ can now be obtained if simple hydrodynamics may be applied to estimate the tumbling correlation time $\tau_{\mathrm{r}}$ and if the mean residence time of a water molecule in the coordination shell of the $\mathrm{Li}^{+}$-cation would be known. Estimates available in the literature give $\tau_{\mathrm{r}}(300 \mathrm{~K})$
$=30 \mathrm{ps}$ [26] and $\tau_{\mathrm{cx}}(300 \mathrm{~K})=14 \mathrm{ps}$ [41] leading to $\tau_{00}=0.15 \mathrm{ps}$, a figure in reasonable agreement with the bestfit value $\tau_{00}=0.16 \mathrm{ps}$ obtained earlier. In fact, $\tau_{00}$ can easily be estimated from $T_{1}$-data in the fast motions regime where the local mode does not contribute significantly to the relaxation.

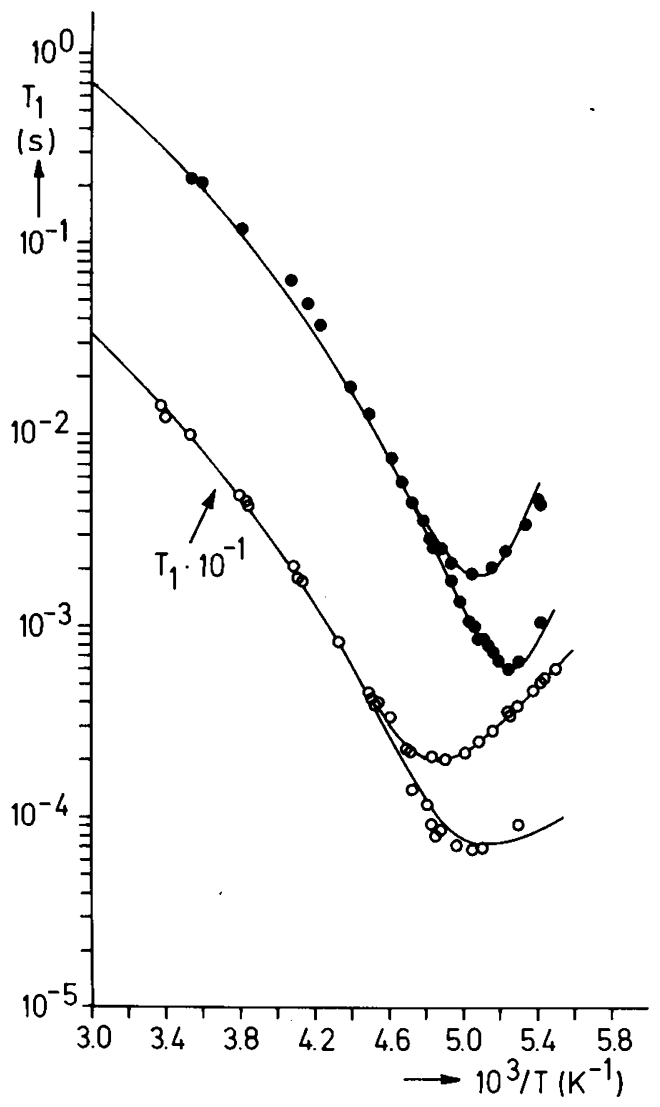

Fig. 2

Deuteron relaxation time curves $T_{1}(T)$ in aqueous LiCl-solutions of composition $C=16.7$ (upper curve) and $C=4.55$ (lower curve). Solid lines represent calculated curves with parameters given in Table 1. The longer (shorter) $T_{1}$ in the slow motions regime (low temperatures) correspond to the higher (lower) ficld $B_{0}=7.05$ Tesla $\left(B_{0}=2.35\right.$ Tesla)

The preexponential factor $\tau_{\mathrm{i} 0}$ and the apparent activation energy $E_{\mathrm{a}}$ for the Arrhenius-law of the local anisotropic mode had been considered freely adjustable parameters previously. But both are highly correlated, hence an infinite number of pairs may lead to equally good fits. This is also indicated by the unsystematic variation of both quantities obtained by least-squares fitting ${ }^{2} \mathrm{H}-T_{1}$ data within the series of alkali-halide/water solutions [42]. A physically more appealing approach is to consider the local anisotropic mode to constitute a thermally activated process. Then $\tau_{\mathrm{i} 0}$ corresponds to an attempt frequency for barrier crossing and is identified with the inverse of the frequency of librations in the energy spectrum of the system. However the librational band is fairly broad with little distinct features in both the Raman- and IR-spectra of aqueous electrolyte solutions [43]. Moreover experimental determinations [44] of the frequency of the band maximum of the hydration water component in aqueous alkali-halide solutions indicate a shift to 
lower frequencies (depending on the concentration) compared to neat water whereas molecular dynamics simulations [13] suggest a displacement by $200 \mathrm{~cm}^{-1}$ to higher frequencies of the primary hydration band maximum for $\mathrm{Li}^{+}$. Anyhow, in view of the oversimplyfied treatment of the local anisotropic mode a mean value of $\lambda_{\max }^{-1}=413 \mathrm{~cm}^{-1}$ corresponding to $\tau_{\mathrm{i} 0}=81 \mathrm{fs}$ may be chosen to reexamine the ${ }^{2} \mathrm{H}-T_{1}$ data obtained at 7.05 Tesla and 2.35 Tesla to deduce the corresponding apparent activation energy $E_{\mathrm{a}}$. Of course, any other choice of $\tau_{i 0}$ will influence the resulting value of $E_{\mathrm{a}}$. The parameters are given in Table 1 and Fig. 2 shows the ${ }^{2} \mathrm{H}$ relaxation time curves for solutions with composition $C=4.55$ and $C=16.67$.

\section{The Two-Site Approximation}

The. $T_{1}(T)$-curves for the more dilute solution have been calculated within the two-site approximation $[2,17]$

$R_{1}(C, T, p)=(n / C)\left(R_{1}(T)\right)_{\text {hyd }}+((C-n) / C)\left(R_{1}(T, p)\right)_{\text {bulk }}$

with an assumed hydration number $n=6$ on which recent neutron-scattering and computer simulation results seem to converge. But with $n=4.55$ a slightly better description of the relaxation time curve is obtained at high temperatures. Around the minimum both curves are virtually indistinguishable. As has been demonstrated earlier [2,17] both rates in Eq. (24) have to be taken at the same reduced temperature $\left(T-T_{0}(C, p)\right)$ as pertains to the actual solution under investigation because the clusters are in dynamic equilibrium. It is for this reason that the physical properties of the bulk water are different from neat water if compared at the same temperature. Also because of the short mean residence time of water molecules beneath a $\mathrm{Cl}^{-}$-anion the influence of the latter on the bulk water dynamics is simply contained in the parameter $T_{0}$ of the system.

The local anisotropic mode contributes to the relaxation time curve $T_{1}(T)$ only at temperatures $T<T_{\min }$ significantly. At these temperatures the tumbling mode slows down strongly and the local mode dominates the relaxation. Thus reliable apparent activation energies $E_{\mathrm{a}}$ may be deduced only if sufficient data at temperatures $T<T_{\min }$ can be collected. For the ${ }^{2} \mathrm{H}-T_{1}$ measurements this was possible only at the higher field $\left(B_{0}=7\right.$ Tesla), because the low temperature limit is set by the deuteron $\mathrm{nmr}$ glass transition where the inverse correlation time $1 / \tau_{\mathrm{r}}$ becomes less than the quadrupole coupling constant. The deuteron spectrum then looks very broad, characteristic of a solid. A slight reduction of the deuterium quadrupole coupling constant $\chi_{\text {eff }}\left({ }^{2} \mathrm{H}\right)$ to 192 $\mathrm{kHz}$ yielded even better agreement at the minimum of the relaxation time curve. Also $\tau_{00}=0.20$ ps results from adjusting the high temperature data.

\section{Estimation of the Parameters for Positional Fluctuations}

In order to use these parameters to calculate the contribution from orientational fluctuations to the ${ }^{1} \mathrm{H}$ and ${ }^{6} \mathrm{Li}$ relaxation rate in $\mathrm{H}_{2} \mathrm{O}$ one must account for the known isotope effect upon librations and the glass temperature $T_{0}$ $[3,18]$. Also the apparent activation energy $E_{\mathrm{a}}$ may differ in light water. It can be extracted from the low temperature data if the contribution to the ${ }^{1} \mathrm{H}$ and ${ }^{6} \mathrm{Li}$ relaxation rates from positional fluctuations can be calculated with Eqs. (13), (14) and (16). The parameters entering these expressions, which have to be known, are the number density $N_{s}$ of interacting spins relaxing the nucleus under consideration. It can be calculated from the mass density of the solution which is known [45]. The distances of closest approach $d_{l-s}$ may be taken from partial radial pair-distribution functions deduced from computer simulations. A value of $d_{1 \mathrm{HI}}=0.225$ $\mathrm{nm}$ and $d_{\mathrm{HLi}}=0.335 \mathrm{~nm}$ will be used as obtained in a MDsimulation of a concentrated $\mathrm{LiCl}-\mathrm{H}_{2} \mathrm{O}$ solution [14]. The latter agrees well with $d_{\mathrm{LiH}}=0.340 \mathrm{~nm}$ as calculated from the radii of the cation and the water molecule. It should be remembered that any pair-correlation effects as well as excentricity effects are neglected in the theory leading to Eq. (15). Consequently the spins are considered to sit in the center of the particles. Nevertheless experimental distances of closest approach between the spin-bearing atoms are used.

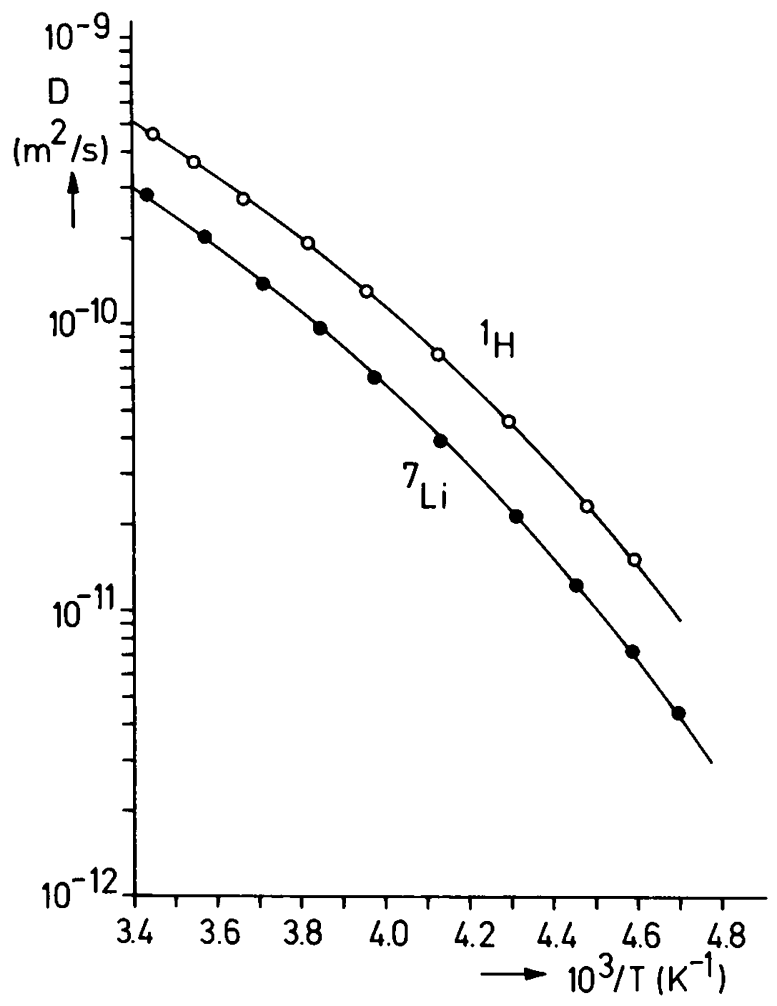

Fig. 3

Proton and lithium self-diffusion cocfficients $D$ versus inverse temperature. Solid line corresponds to a VTF-type temperature dependence. Best fit parameters $D_{0}$ and $B_{\mathrm{p}}$ are given in Table 1

\section{Self-Diffusion of Protons and $\mathrm{Li}^{+}$-Cations}

The self-diffusion coefficients of the $\mathrm{Li}^{+}$-cations and of the protons entering the spectral density functions (Eqs. (13) -(16)) could be measured to $213 \mathrm{~K}$. The experimental data are shown in Fig. 3. If they are plotted in a modified Arrhenius diagram versus $\left(T-T_{0}\right)$ with $T_{0}=133 \mathrm{~K}$ as predicted according to the general rule discussed above, straight lines are obtained justifying the assumption of a VTF-type temperature dependence as for the tumbling 
mode. This pronounced non-Arrhenius behaviour is in clear contradiction to assertions made earlier $[32,33]$ and invalidates conclusions drawn thereoff about the mobility of water molecules at the glass-transition.

The self-diffusion coefficient of water protons $D(1 \mathrm{H})$ is larger and possesses a slightly weaker temperature dependence than the self-diffusion coefficient of the $D\left(\mathrm{Li}^{+}\right)$. This implies a larger translational mobility of water protons and a stronger slowing down of the mobility of $\mathrm{Li}^{+}$-cations. At first sight this observation seems to jeopardize the assumption of a well defined hydration shell of the cations with $\tau_{\mathrm{cx}}$ at least of the order of $\tau_{\mathrm{d}}, \tau_{\mathrm{r}}$ as well as the assertion of a close correspondence of local structures in the glassy phase and in the undercooled liquid phase $[3,10,46]$. But a higher proton mobility compared with the diffusivity of the cations may arise in two different ways. Either are the positional fluctuations of the $\mathrm{Li}^{+}$-ions and their surrounding water molecules largely uncorrelated because of short mean residence times of the water molecules in the hydration shell or the protons possess an extra freedom to migrate due to short mean lifetimes within a water molecule.

The former possibility would imply $\tau_{\text {cx }} \ll \tau_{\mathrm{d}}$ (ion) $=35$ ps at $T=300 \mathrm{~K}$. Note, however, that a study of the cationic diffusivity of $\mathrm{LiNO}_{3}$ dissolved in liquid ammonia, a strongly polar solvent with greatly diminished $\mathrm{H}$-bonding abilities, showed $D\left(\mathrm{Li}^{+}\right)=D\left(\mathrm{NH}_{3}\right)$ above 20 mole- $\%$ metal [47]

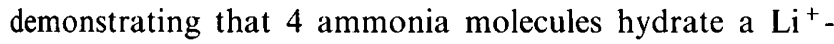
cation and diffuse with it because of a long enough mean residence time. Although $\tau_{\mathrm{cx}}$ is not really known, estimates exist in the literature. A MD-simulation of $\mathrm{Li}^{+}+64 \mathrm{H}_{2} \mathrm{O}$ obtaines $\tau_{\mathrm{cx}}=30$ ps [26], Okada et al. [49] simulated the system $\mathrm{Li}^{+}+4 \mathrm{H}_{2} \mathrm{O}$ and calculated the mean exchange velocity $v=7 \mathrm{~m} / \mathrm{s}$. With a distance $r=0.246 \mathrm{~nm}$ a mean residence time of $35 \mathrm{ps}$ is obtained at $T=300 \mathrm{~K}$. Also Friedman et al. [34] estimate $\tau_{\mathrm{cx}}=14 \mathrm{ps}$ at $T=298 \mathrm{~K}$. These estimates show that $\tau_{\mathrm{cx}}$ is certainly of the order of $\tau_{\mathrm{d}}$ or $\tau_{\mathrm{r}}$ as assumed.

An extra proton mobility over that of the water molecules might arise because of the strong polarization of the $\mathrm{OH}$ bond of a water molecule in the $\mathrm{Li}^{+}\left(\mathrm{H}_{2} \mathrm{O}\right)_{4} \mathrm{Cl}^{-}$clusters, leading to a large hydrolysis constant invoked recently to explain the $p, T$-dependence of the conductivity in concentrated $\mathrm{LiCl}-\mathrm{H}_{2} \mathrm{O}$ solutions [48]. In this context it is interesting that the postulated excess proton mobility was envisaged to depend on the local anisotropic reorientation of a water molecule in the hydration sphere. As this mode possesses a much weaker temperature dependence at low temperatures one might conclude that the proton diffusivity will also exhibit a weaker temperature dependence than the ionic diffusivity. The latter should be determined largely by the configurational fluctuations associated with the glasstransition, hence the VTF-parameters should be similar to those found for the tumbling mode. This is corroborated by the best-fit parameters collected in Table 1. If $B_{\mathrm{p}}(\mathrm{Li})$ is given the value of $B_{\mathrm{r}}$ an almost equally good description of the temperature dependence of the cationic diffusion coefficient results.

With these ingredients Eqs. (13) - (16) have been used to calculate the contribution from positional fluctuations to the ${ }^{1} \mathrm{H}$ and ${ }^{6} \mathrm{Li}$ relaxation rates. The analogous determination of the contribution from orientational fluctuations with Eqs. (4) -(12) requires knowledge of the internuclear distances $r_{\mathrm{HH}}$ and $r_{\mathrm{HLI}}$. Both have been taken from neutron scattering data [6], although it should be noted that X-ray and computer simulations give somewhat larger distances [14]. The good agreement between the calculated and the experimental relaxation rates for both ${ }^{1} \mathrm{H}$ and ${ }^{6} \mathrm{Li}$ nuclei (see Fig. 4) thus confirms the neutron scattering results. Last, it turned out that the apparent activation energy $E_{\mathrm{a}}$ of the local anisotropic mode had to be adjusted to obtain the right slope of the relaxation time curves at low temperatures. The adjustment had been made with the ${ }^{1} \mathrm{H}-T_{1}$ data alone. The activation energy is slightly smaller in $\mathrm{H}_{2} \mathrm{O}$ than in $\mathrm{D}_{2} \mathrm{O}$. This might be due to the weaker $\mathrm{H}$-bond strength in light water, although hydrogen bonding is certainly not extensive in this system. The deviation of the longest ${ }^{6} \mathrm{Li}-T_{1}$ from the calculated curve may arise from small amounts of paramagnetic impurities.

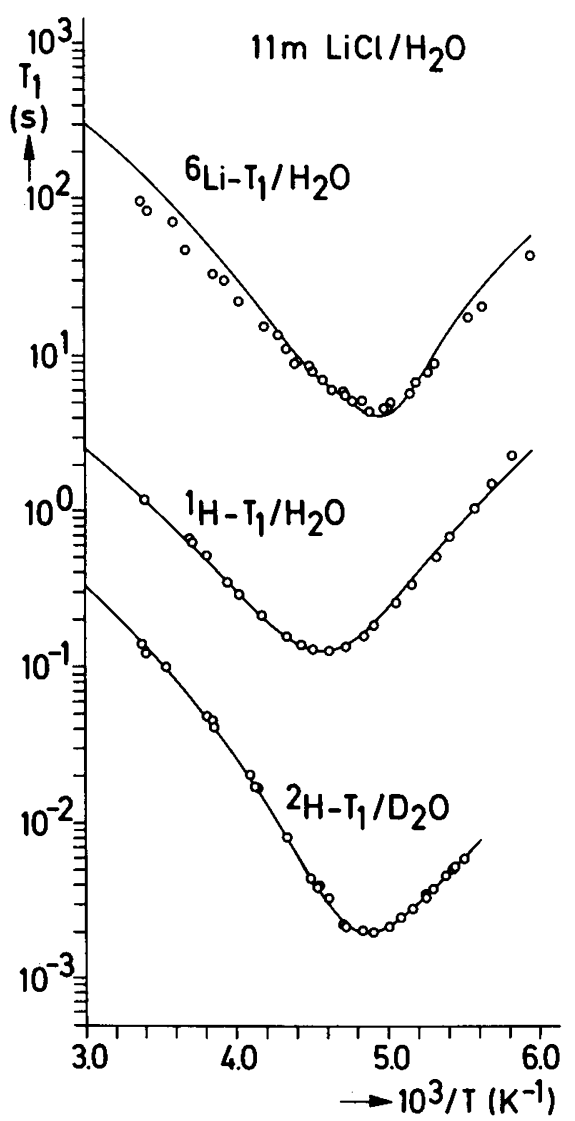

Fig. 4

Relaxation time curves $T_{1}\left(T, B_{0}=7\right.$ Tesla) of ${ }^{1} \mathrm{H}$ and ${ }^{6} \mathrm{Li}$-nuclei in $11 \mathrm{~m} \mathrm{LiCl} / \mathrm{H}_{2} \mathrm{O}$ solutions and of ${ }^{2} \mathrm{H}$ nuclei in $11 \mathrm{~m} \mathrm{LiCl} / \mathrm{D}_{2} \mathrm{O}$ solutions. Solid lines represent calculated curves with parameters given in Table 1

\section{Conclusions}

A two-mode approximation for orientational fluctuations and experimentally determined translational diffusivities prooved adequate to describe the relaxation time curves of the various nuclei $\left({ }^{2} \mathrm{H},{ }^{1} \mathrm{H},{ }^{6} \mathrm{Li}\right)$ extending well into the slow motions regime near the glass-transition. Hence the dynamical model, proposed recently, is internally consistent and corroborates the average local structure of the 
$\mathrm{Li}^{+}\left(\mathrm{H}_{2} \mathrm{O}\right)_{n} \mathrm{Cl}^{-}$clusters as deduced from NMR and Neutron scattering experiments. It incorporates the strongly non-Arrhenian $T$-dependence of dynamic properties at medium undercooling and the return to an Arrhenius behaviour at strong undercooling in terms of a VTF-type behaviour of global structural rearrangements in contrast to an Arrhenius behaviour of thermally activated local modes dominating the relaxation near the glass-transition. But the dynamical model with its current parameterization fails to reproduce the relaxation time curve of ${ }^{6} \mathrm{Li}$ in $\mathrm{LiCl} / \mathrm{D}_{2} \mathrm{O}$ in the slow motions regime. The model predicts a further minimum due to the local anisotropic mode not seen experimentally. This points to a deficiency of the spectral density function and calls for a more elaborate treatment of the local anisotropic fluctuations. However the very weak spin-lattice interactions (note the extremely long relaxation times) render this system potentially sensible to artefacts due to a contamination with paramagnetic impurities. Further experiments at different frequencies, currently under way in our laboratory, are necessary to resolve that issue and to supply further information as to the form of the spectral density functions involved. This is also obvious from the mere fact that the two earlier investigations of this system could describe their data with two motional models with distinctly different frequency dependences in the slow motions regime.

We are indebted to Prof. Lüdemann for stimulating discussions. The expert technical help of $\mathrm{H}$. Knott, S. Heyn and E. Treml made this work feasible. Support from the DFG and the Fonds der Chemie is greatfully acknowledged.

\section{References}

[1] E. W. Lang, W. Fink, and H.-D. Lüdemann, J. Phys. (Paris) 45, C7-173 (1984).

[2] E. W. Lang and H.-D. Lüdemann, Ber. Bunsenges. Phys. Chem. 89, 508 (1985).

[3] E. W. Lang and L. Piculell, in: "Water and Aqueous Solutions", eds. G. W. Neilson and J. E. Enderby, Hilger, Bristol 1986.

[4] P. Laszlo, in: "The Multinuclear Approach to NMR Spectroscopy", eds. J. B. Lambert and F. G. Riddell, Reidel, Dordrecht 1983

[5] F. X. Prielmeier, R. J. Speedy, and H.-D. Lüdemann, Proc. XIth AIRAPT Conference, Kiew 1987, in press.

[6] G. W. Neilson, in: "Water and Aqueous Solutions", eds. G. W. Neilson and J. E. Enderby, Hilger, Bristol 1986.

[7] A. H. Narten, F. Vaslow, and H. A. Levy, J. Chem. Phys. 58, 5017 (1973).

[8] J. R. Newsome, G. W. Neilson, and J. E. Enderby, J. Phys. C: Solid State Phys. 13, L923 (1980).

[9] A. P. Copestake, G. W. Neilson, and J. E. Enderby, J. Phys. C: Solid State Phys. 18, 4211 (1985).

[10] I. C. Baianu, N. Boden, D. Lightowlers, and M. Mortimer, Chem. Phys. Lett. 54, 169 (1978).

[11] A. Geiger and H. G. Hertz, J. Solut Chem. 5, 365 (1976).
[12] R. Mazitov, K. J. Müller, and H. G. Hertz, Z. Phys. Chem. Neue Folge 140, 55 (1984).

[13] K. Heinzinger, Physica 131 B, 196 (1985).

[14] P. Bopp, I. Okada, H. Ohtaki, and K. Heinzinger, Z. Naturforsch. $40 a, 116$ (1985).

[15] M. F. Mills, J. R. Reimers, and R. O. Watts, Mol. Phys. 57. 777 (1986).

[16] F. T. Marchese and D. L. Beveridge, J. Am. Chem. Soc. 106. 3713 (1984).

[17] N. Boden and M. Mortimer, J. Chem. Soc. Faraday Trans. II, 74, 353 (1978).

[18] E. W. Lang, H.-D. Lüdemann, and L. Piculell, J. Chem. Phys. 81,3820 (1984).

[19] H. Wennerström, Mol. Phys. 24, 69 (1972).

[20] B. Halle, Mol. Phys. 53, 1427 (1984).

[21] U. Bengtzelius, W. Götzc, and A. Sjölander, J. Phys. C: Solid State Phys. 17, 5915 (1984).

[22] J. Jäckle, Rep. Prog. Phys. 49, 171 (1986).

[23] K. L. Ngai, Comments Solid State Phys. 9, 127 (1979).

[24] F. W. Wehrli, J. Magn. Reson. 23, 527 (1976).

[25] B. P. Fabricand and S. S. Goldberg, Mol. Phys. 13, 323 (1967).

[26] R. W. Impey, P. A. Madden, and I. R. McDonald, J. Phys. Chem. 87, 5071 (1983).

[27] L.-P. Hwang and J. H. Freed, J. Chem. Phys. 63. 4017 (1975).

[28] Y. Ayant, E. Belorizky, J. Alizon, and J. Gallice, J. Phys. (Paris) 36, 991 (1975)

[29] J. P. Albrand, M. C. Taieb, P. H. Fries, and E. Belorizky, J. Chem. Phys. 78, 5809 (1983).

[30] P. H. Fries, Mol. Phys. 48, 503 (1983)

[31] E. W. Lang and H.-D. Lüdemann, Angew. Chem. Int. Ed. Engl. 21, 315 (1982).

[32] E. J. Sutter and J. F. Harmon, J. Phys. Chem. 79, 1958 (1975).

[33] J. F. Harmon and E. J. Sutter, J. Phys. Chem. 82, 1938 (1978).

[34] H. L. Friedman and L. Lewis, J. Solut. Chem. 5, 445 (1976).

[35] S. A. Brawer, J. Chem. Phys. 81, 954 (1984).

[36] H. Kanno, I. Shirotani, and S. Minomura, Bull. Chem. Soc. Jpn. 53, 2079 (1980).

[37] H. Weingärtner, J. Magn. Reson. 41, 74 (1980)

[38] S. Engström, B. Jönsson, and R. W. Impey, J. Chem. Phys. 80,5481 (1984).

[39] S. Engström, B. Jönsson, and B. Jönsson, J. Magn. Reson. 50. 1 (1982)

[40] E. W. Lang, in preparation.

[41] J. P. Hunt and H. L. Friedman, Prog. Inorg. Chem. 30, 359 (1983).

[42] W. Fink, Dissertation, University of Regensburg 1987.

[43] T. H. Lilley, in: "Water - A Comprehensive Treatise", Vol. 3, p. 265ff, ed. F. Franks, Plenum Press, New York 1973.

[44] D. W. James and R. F. Armishaw, Aust. J. Chem. 28, 1179 (1975).

[45] C. T. Moynihan, N. Balitactac, L. Boone, and T. A. Litovitz, J. Chem. Phys. 55, 3013 (1971).

[46] M. Goldstein, Faraday Symp. Chem. Soc. 6, 7 (1972).

[47] R. Kusaka, K. Ban, Y. Nakamura, and S. Shimokawa, J. Phys. Chem. 91, 985 (1987).

[48] D. R. MacFarlane, J. Scheirer, and S. I. Smedley, J. Phys. Chem. 90, 2168 (1986).

[49] I. Okada, Y. Kitsumo, H. Lee, and H. Ohtaki, in: "Ions and Molecules in Solution", eds. N. Tanaka, H. Ohtaki, and R. Tamamushi, Elsevier, Amsterdam 1983.

(Eingegangen am 14. Januar 1988, 